\title{
Case Report: Ascending Myelo-Encephalitis after a Penetrating Injury to the Foot: An Atypical Case of Neuromelioidosis
}

\author{
Boby Varkey Maramattom ${ }^{1}$ and Balram Rathish ${ }^{2 *}$ \\ ${ }^{1}$ Department of Neurology, Aster Medcity, Kochi, India; ${ }^{2}$ Department of Internal Medicine, Aster Medcity, Kochi, India
}

\begin{abstract}
Organisms penetrate the central nervous system (CNS) via three routes. The commonest is the hematogenous route, and other routes include contiguous or penetrating injury or rarely via retrograde axoplasmic route. Although the axoplasmic highway is often used by viruses, only a few bacteria are known to penetrate the CNS via this route. We present a 57-year-old man who developed a penetrating injury while working in a field. Over the next 4 months, he developed pain at the site of the poorly healing wound, which ascended up the right leg and presented as a conuscauda syndrome. Magnetic resonance imaging (MRI) showed an enhancing intradural intramedullary enhancing lesion in the conus on the right side with cord edema from D11 to L1 level. Extensive evaluation was negative, and he continued to progress to holocord myelitis and developed bilateral corticospinal tract lesions ("tractopathy") in the brain stem and internal capsule. He died after developing a right-sided cerebritis with mass effect. Tissue biopsy from the brain at the time of decompressive craniectomy grew Burkholderia pseudomallei and confirmed a diagnosis of neuromelioidosis (NM). We reviewed the literature for $\mathrm{NM}$, its variable presentations, and the concept of an "infectious tractopathy" and imaging findings which could generate suspicion of this entity.
\end{abstract}

\section{INTRODUCTION}

Many organisms penetrate the central nervous system (CNS) using the retrograde axoplasmic transport mechanism (Table 1). A notable new entrant in the list is SARSCoV-2, the virus responsible for the present pandemic. However, only a few bacteria spread via this mechanism. We encountered a patient who in retrospect seemed to have contracted a myelitis which ascended via retrograde transport from a limb injury through the sciatic nerve (peripheral nervous system). The myelitis rapidly extended up from the conus to the cervical spinal cord, brain stem, and then to the cerebrum via the corticospinal tracts (CSTs). We searched the literature for similar cases and present a literature review of atypical presentations of NM and infective "tractopathies."

\section{CASE REPORT}

A 57-year-old previously healthy man developed acute onset of weakness in his right leg of 2 days duration. Four months previously, he had sustained a penetrating injury to his right foot while working in a waterlogged field. His right foot had become swollen and painful. He underwent wound debridement 11 days after sustaining the injury, and a retained foreign body was retrieved. Over the next 45 days, his pain increased and ascended from his right foot to the right knee and lower back. The patient repeatedly complained that his symptoms had gradually ascended from his leg to his back. An arterial doppler ruled out peripheral vascular disease as the cause of delayed wound healing. Spinal MRI showed an intradural intramedullary enhancing lesion in the conus on the right side with cord edema and expansion from D11 to L1 level (Figure 1). MRI brain was normal at the time. Chest X-ray was normal. CSF study revealed a high cell count of 600 cells $/ \mathrm{cmm}^{3}$ (N3L97),

*Address correspondence to Balram Rathish, Department of Internal Medicine, Aster Medcity, Cheranalloor P.O. Kochi 682027, India. E-mail: balramrnair@gmail.com
TABLE 1

Neuroinvasive organisms that use axoplasmic transport mechanisms to penetrate the central nervous system. ${ }^{19-23}$

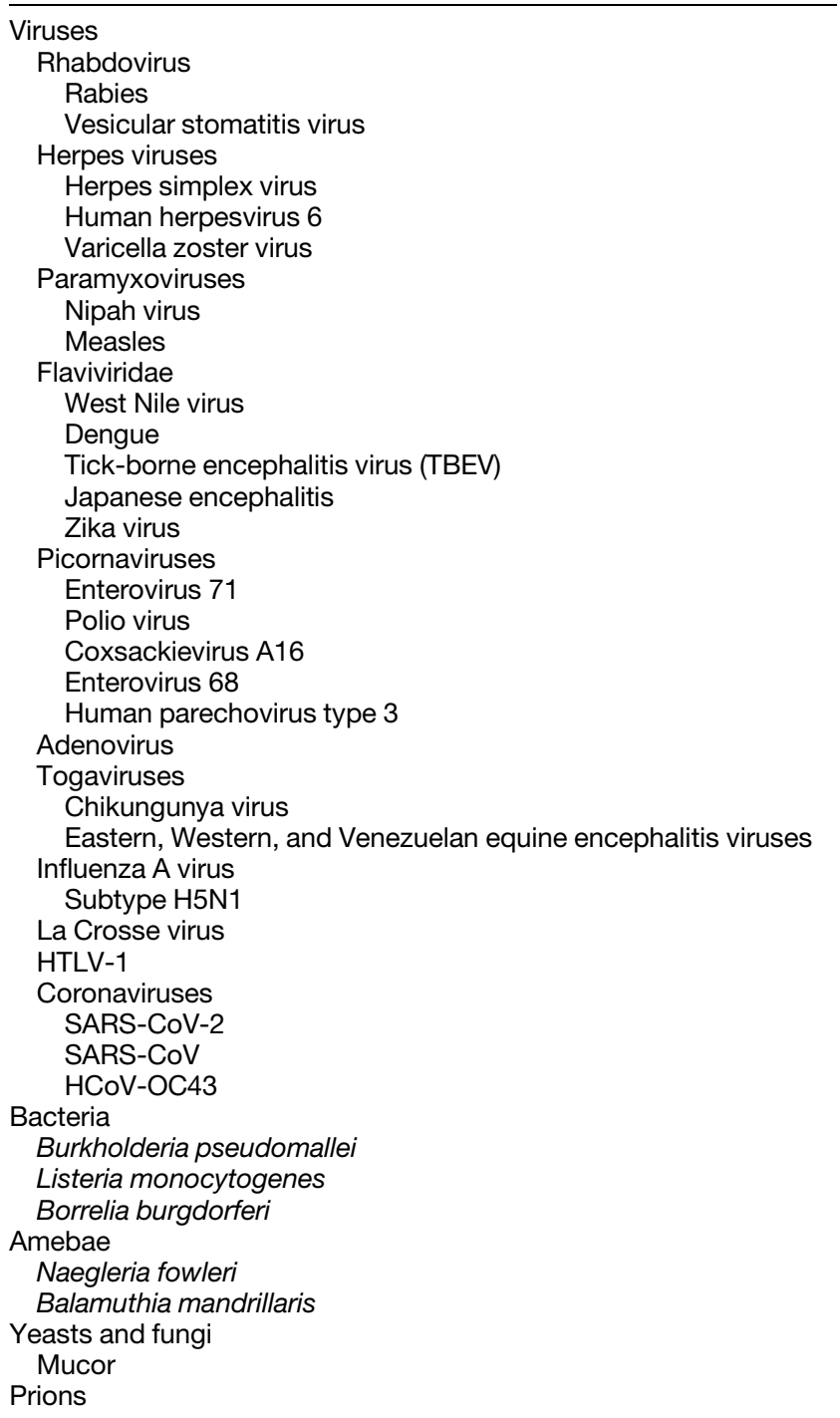



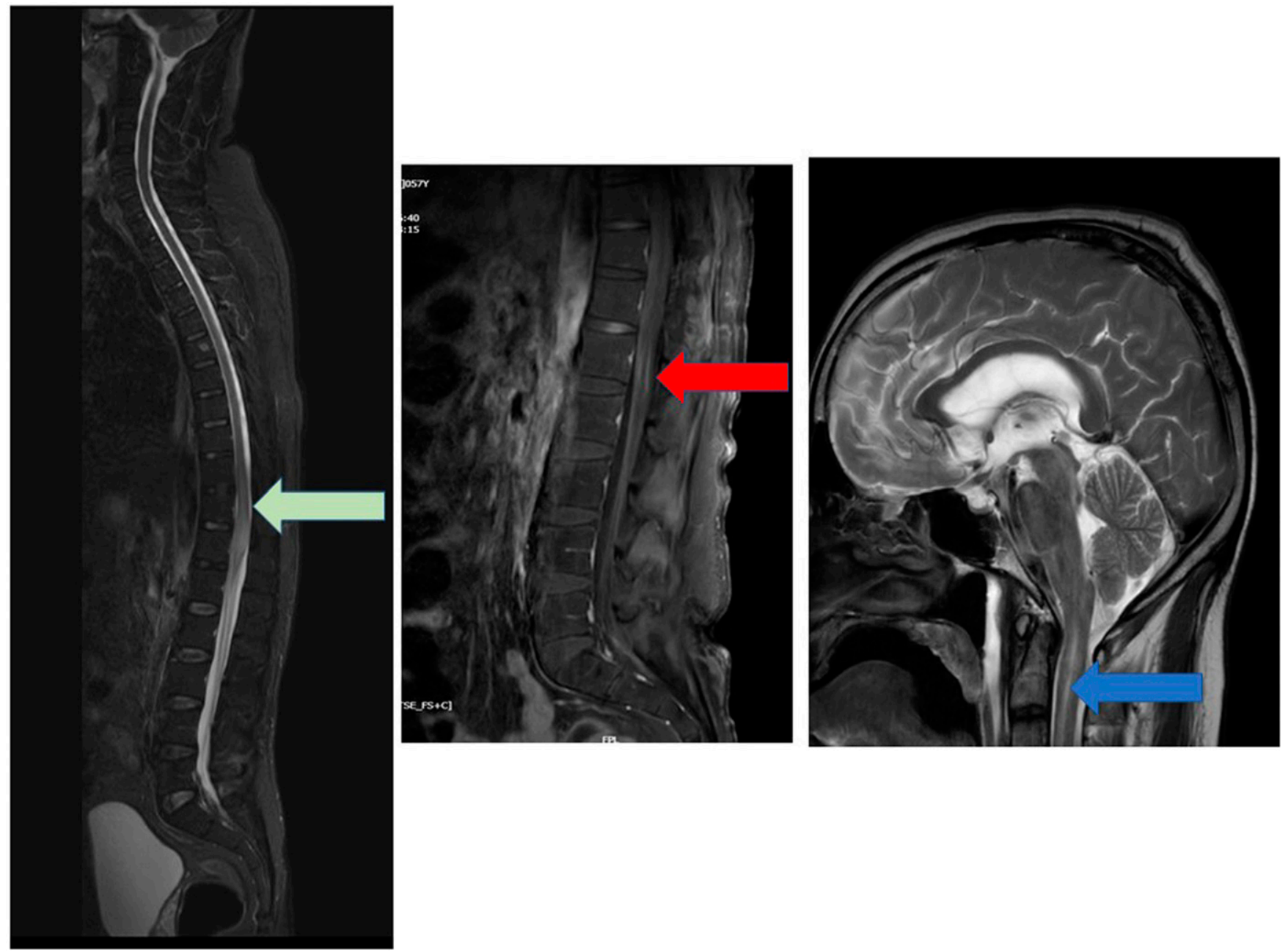

FIGURE 1. Left panel. Sagittal T2-weighted image. Green arrow shows the conus myelitis. Middle panel. Red arrow shows the leptomeningeal enhancement of the conus. Right panel. Blue arrow shows upper cervical cord long segment hyperintensities. This figure appears in color at www.ajtmh.org.

normal sugar levels, and high protein (900 mg\%). A multiplex PCR panel (XCyton SES-Meningitis panel) was positive for Bacteroides fragilis. The initial possibilities were infective myelitis or an inflammatory demyelinating disease.

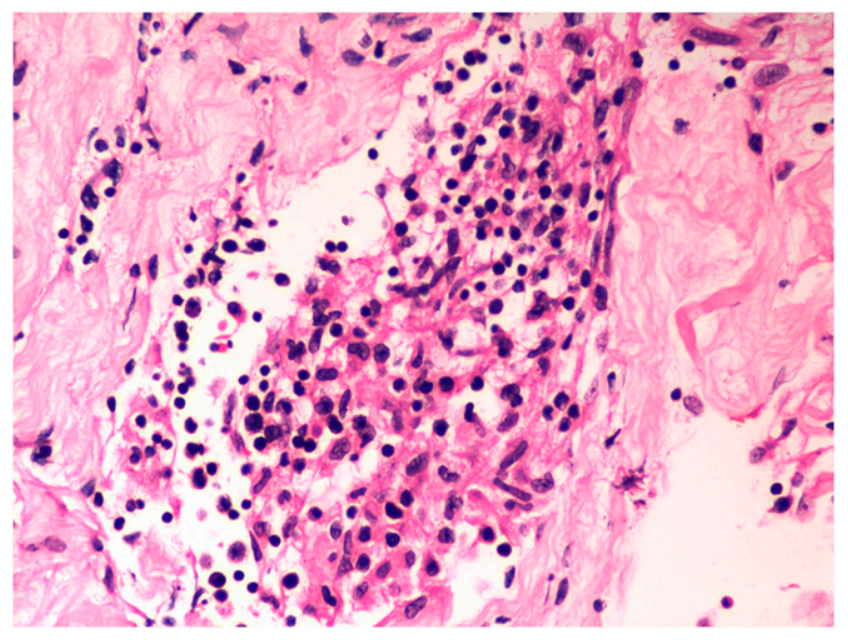

FIGURE 2. Histopathology of spinal cord biopsy showing a noncaseating granuloma. This figure appears in color at www.ajtmh.org.
CSF malignant cytology, antibodies for neuromyelitis optica spectrum disorders, tuberculosis, vasculitic panels, VDRL, TPHA, blood, and urine cultures, and CSF for West Nile virus, Japanese encephalitis, and Zika virus were negative. In 5 days, he developed complete paraplegia with bowel and bladder retention and a D6 sensory level. On day 7 , a spinal cord and nerve root biopsy were performed which showed noncaseating granulomas on histopathology, was sterile on microbiological culturing with prolonged incubation, and was negative for acid-fast bacilli on ZiehlNeelsen staining, GeneXpert, and mycobacterial culturing (Figure 2). Fungal cultures were negative. Because of the presence of noncaseating granulomas, neurosarcoidosis was considered, and intravenous methylprednisolone (IVMP) $1 \mathrm{gm} /$ day was coadministered for 5 days along with ceftazidime and vancomycin for 2 weeks. He showed some symptomatic improvement, and his sensory level came down to D10 level.

He was transferred for rehabilitation, where he developed a high-grade fever and altered mental status 3 days later. Weakness was gradually ascending upward to a quadriparesis. On day 26, MRI showed hyperintensities extending upward from the conus to the cervical spine as well as new FLAIR hyperintensities along the CST in the pons and both 


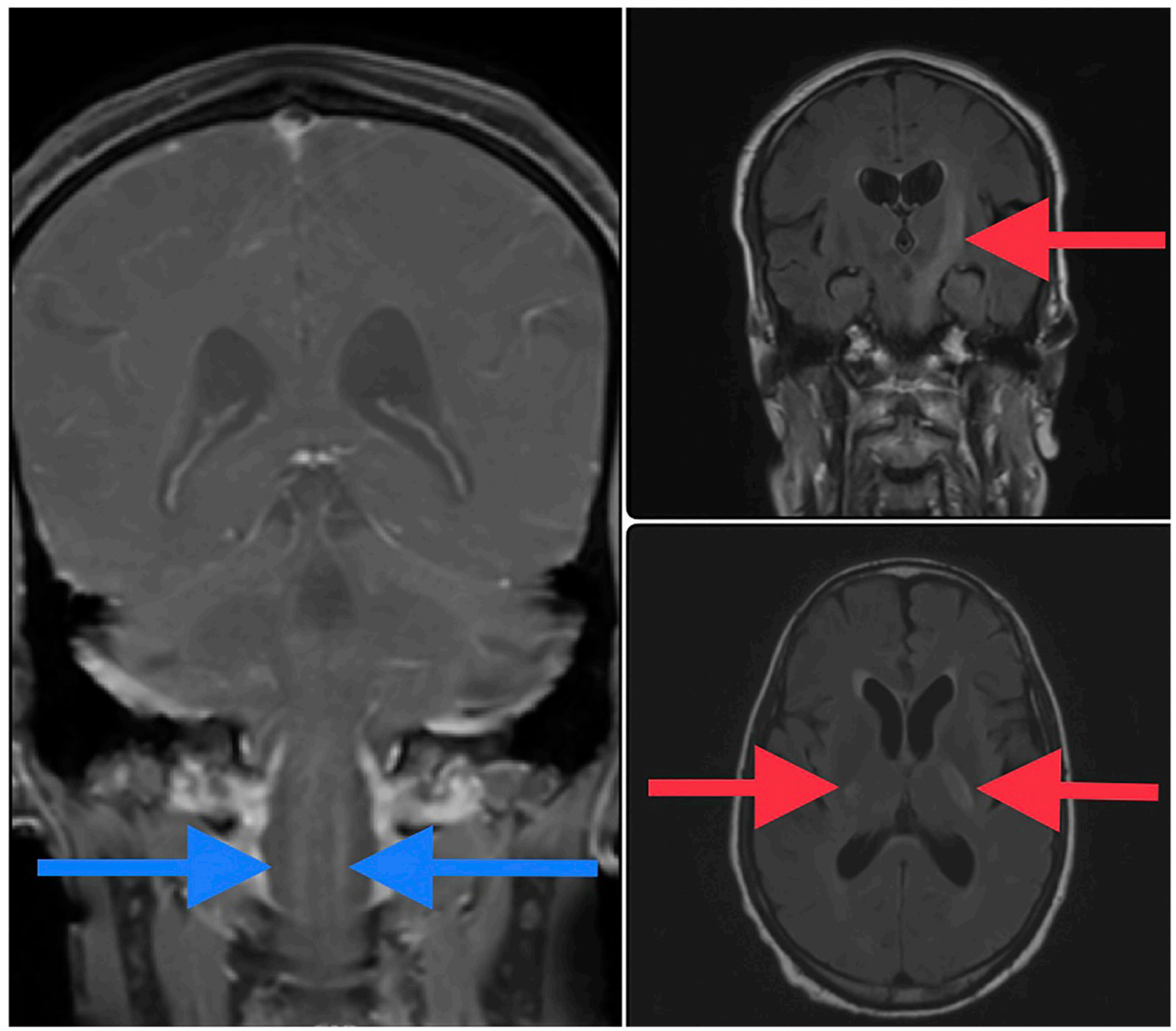

FIGURE 3. Left panel. Coronal T1 contrast MRI shows the linear corticospinal tract (CST) enhancing tractopathy (blue arrows). Right upper panel. Red arrow shows the left CST hyperintensity on FLAIR coronal image. Right lower panel. Red arrows show bilateral internal capsular FLAIR hyperintensities. This figure appears in color at www.ajtmh.org.

internal capsules (left $>$ right) (Figure 3). Meropenem 2 gm thrice daily and vancomycin $900 \mathrm{mg}$ thrice daily were started, along with intravenous immunoglobulin (IVIG) $2 \mathrm{gm} / \mathrm{kg}$ and intravenous methylprednisolone (IVMP) $1 \mathrm{gm} /$ day, suspecting a possible reactivation of the infection, immune reconstitution inflammatory syndrome, or a postinfectious acute disseminated encephalomyelitis. However, on day 40 , computed tomography brain showed right hemispheric edema with midline shift. An emergency decompressive craniectomy, and leptomeningeal and brain biopsy were performed. He expired 2 days later (day 42). The family refused an autopsy because of religious reasons. Neuropathology showed parenchyma infiltrated with neutrophils and histiocytes and multifocal infiltrates with microabscesses (Figure 4). Microbial elements, granulomas, or neoplastic cells were absent. The features were suggestive of a suppurative inflammatory lesion. The tissue culture grew Burkholderia pseudomallei 3 days later, and confirmation was received from the reference laboratory 10 days later (Figure 5). The organism was identified by biochemical tests and Vitek2 at our center, as well as confirmation by monoclonal antibody-based latex agglutination for B. pseudomallei, Active Melioidosis DetectTM rapid test (InBios, Seattle, WA), and molecular identification based on T3SS1 gene from a reference laboratory.

\section{DISCUSSION}

Melioidosis (Whitmore's disease, "Vietnamese time bomb") is caused by the Gram-negative environmental bacterium $B$. pseudomallei, a saprophyte found in the soil, 


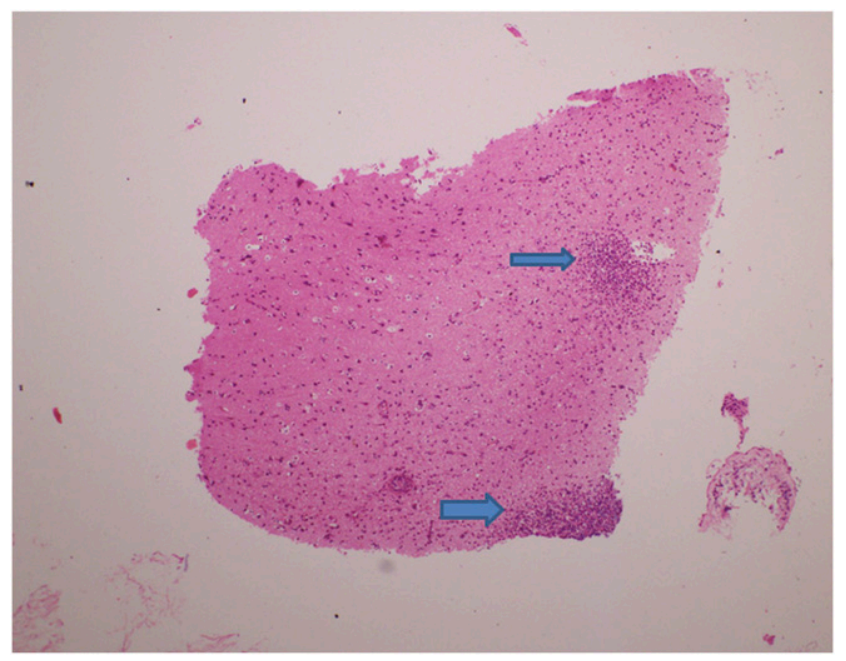

FIgURE 4. Histopathology of brain tissue biopsy showing multifocal infiltrates with microabscesses (blue arrows). This figure appears in color at www.ajtmh.org.

rice paddies, and ponds mainly in South East Asia and Australian region but with recent reports from a wider geographical region. ${ }^{1,2}$ Melioidosis is derived from the terms "melis" and "eidos" meaning "distemper of asses" and "resemblance," respectively. ${ }^{1}$ "This is because systemic melioidosis (SM) is often associated with multiple tissue abscesses and resembles glanders, a disease of equines caused by $B$. mallei. Although neuromelioidosis (NM) is rarer than SM, it has a mortality rate of around $30 \%$ with residual neuro-deficits in $50 \%$ of patients. ${ }^{2}$

Burkholderia pseudomallei enters the human host through ingestion, inoculation, or inhalation. Central nervous system penetration occurs via the three main routes: the hematogenous route, direct inoculation, or axoplasmic transport mechanism (via peripheral or cranial nerves). The "trigeminal highway" into the rhombencephalon (via trigeminal innervation of the nasal cavity) is already well known. ${ }^{3}$

Many organisms use the axoplasmic transport mechanism to invade the CNS via the cranial or peripheral nerves,

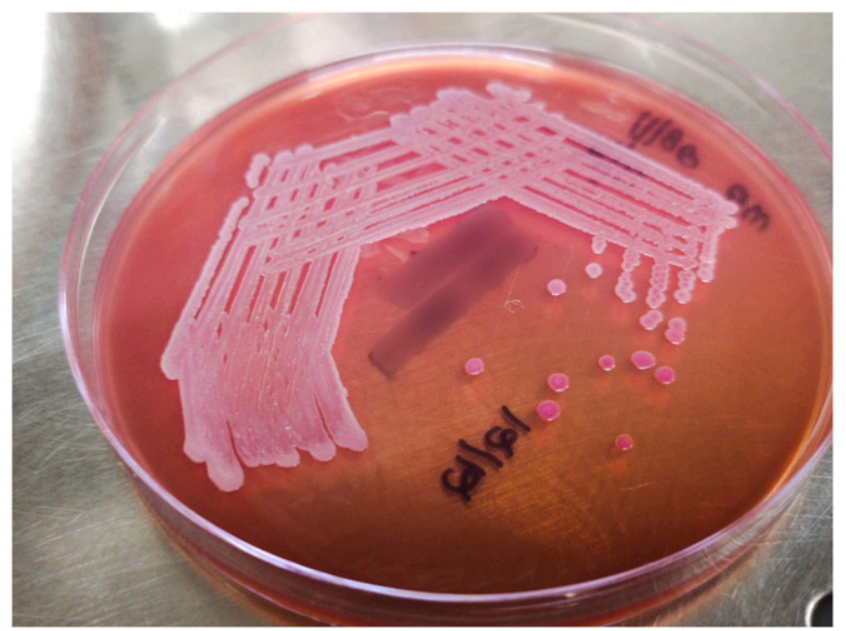

FIGURE 5. Blood agar plate showing small, smooth, cream-colored colonies with a metallic sheen suggestive of Burkholderia pseudomallei. This figure appears in color at www.ajtmh.org. especially viruses which "hitchhike" onto the microtubular assembly of axons for retrograde transport. An archetypal example is the rabies virus (Table 1$).{ }^{4}$ Few bacteria use this mode of transport, with hematogenous being the most common mode of entry into the CNS. However, Listeria and $B$. pseudomallei can migrate upward in the intra-axonal compartment. This intra-axonal movement and intracellular confinement within the parenchyma (sequestration) reduce CSF spillover (explaining the low rate of isolation of these organisms from CSF). This compartmentalization also helps it to bypass the blood-brain barrier. ${ }^{2}$

Clinical manifestations of NM include aseptic meningitis, brain stem encephalitis, hemi- or quadriparesis, myelitis, encephalomyelitis, monoparesis, paraparesis, cranial nerve palsies, or Guillain-Barre syndrome. ${ }^{5}$ Systemic manifestations such as concurrent lung involvement are seen in more than $50 \%$ of patients, and overlying osteomyelitis of the skull or a scalp abscess is found in more than $25 \%$ of patients. ${ }^{6}$ Isolated NM is more difficult to diagnose, and although the CSF shows pleocytosis, high protein, and reduced glucose, CSF studies rarely isolate the organism (because of sequestration). Repeated tissue biopsy and culture are often required to confirm the diagnosis. ${ }^{7} \mathrm{Nev}-$ ertheless, the finding of noncaseating granuloma should prompt one to consider NM in the differential diagnosis. In our patient, the identification of the granuloma on histopathology of spinal biopsy made the likelihood of a viral etiology such as rabies infection or poliomyelitis unlikely. The absence of a history of exposure to animal bite or scratches also made rabies unlikely.

MRI may show multiple scattered microabscesses in the cerebrum, brain stem, cerebellum, or spinal cord with flaky, ring-enhancing, or linear enhancement. Enhancement of the trigeminal nerves with contiguous spread to brain stem trigeminal nuclei can be seen as also overlying osteomyelitis of the skull with a contiguous spread. ${ }^{8}$ As seen in our patient, the ascending tracts such as the CST are often involved. When projection fibers, and long and short association fibers are involved, a "tractopathic" lesion can be discerned on MRI and should arouse the suspicion of an infective tractopathy with an organism that migrates along axonal tracts such as herpes simplex virus, West Nile virus, poliomyelitis, tick-borne encephalitis, Listeria, or B. pseudomallei. ${ }^{9,10}$

Antibiotic therapy for NM includes parenteral ceftazidime or carbapenem plus co-trimoxazole for 4 weeks, followed by oral therapy with co-trimoxazole for a minimum of 3 months. ${ }^{7,11}$ Recurrent melioidosis occurs with noncompliance or incomplete treatment but can also occur after apparent cure ${ }^{2}$; $75 \%$ of recurrences are due to original strain and occur within a year of the initial episode, whereas $25 \%$ of recurrences are due to reinfection and occur later than 1 year 12. In our patient, the initial CSF multiplex PCR revealed the presence of $B$. fragilis, which was in all likelihood a contaminant as the clinical syndrome did not fit. Despite this, as no other diagnosis was readily evident, we treated the patient with IV ceftazidime, which in most likelihood inadvertently treated melioidosis. The good clinical response to ceftazidime was also unfortunately attributed to IVMP and IVIG. Once the antibiotic was stopped, he showed clinical worsening, and by the time meropenem was started, his clinical condition had worsened considerably. 
Inoculation through minor foot wounds is a major mode of acquisition for melioidosis. The incubation period varies from 1 day to 2 months. However, a "tractopathic" spread of NM from peripheral nerves has not yet been described. ${ }^{12}$ McLeod et al. ${ }^{13}$ reported an 8-year-od boy with myelitis and a residual foot drop with a slow-healing skin lesion on the thigh of the most affected side. They did speculate about the possibility of a spinal cord infection via retrograde axoplasmic transport of $B$. pseudomallei from the skin lesion; however, MRI or CSF correlation was not available for review. In another report, MRI showed a similar spinal cord ascending myelitis from the conus to the cervico-medullary junction on MRI. However only T2-weighted images were available, and the lesion was thought to be para-infectious transverse myelitis, even though CSF grew $B$. pseudomallei and the possibility of an infectious tractopathy was not considered. ${ }^{14}$

Strangely, the patient had repeatedly reminded us that his problem had started from his foot injury and ascended up. In retrospect, this is similar to paresthesia at the site of the bite at the onset of rabies. ${ }^{15}$ Unfortunately, we could not obtain an autopsy and a detailed neuropathological study of the brain and spinal cord. Nevertheless, the inoculation mode, lengthy incubation period, and subsequent CST "tractopathic" spread are compatible with this mode of spread. We postulate that linear enhancing hyperintensities along specific tracts or microabscesses along tracts should arouse the suspicion of an infectious "tractopathy" in the appropriate setting, and there should be a high degree of suspicion for neuromeliodosis. ${ }^{16,17,18}$

Received June 6, 2020. Accepted for publication November 25, 2020. Published online January 11, 2021.

Acknowledgment: The American Society of Tropical Medicine and Hygiene (ASTMH) assisted with publication expenses.

Authors' addresses: Boby Varkey Maramattom, Department of Neurology, Kochi, India, E-mail: bobvarkey@gmail.com. Balram Rathish, Department of Internal Medicine, Kochi, India, E-mail: balramrnair@ gmail.com.

\section{REFERENCES}

1. Stanton AT, Fletcher W, 1921. Melioidosis, a new disease of the tropics. Trans Fourth Congr Far East Assoc Trop Med 2: 196-198.

2. Currie BJ, Ward L, Cheng AC, 2010. The epidemiology and clinical spectrum of melioidosis: 540 cases from the 20 year DARWIN prospective study. PLoS Negl Trop Dis 4: e900.

3. St John JA et al., 2014. Burkholderia pseudomallei penetrates the brain via destruction of the olfactory and trigeminal nerves: implications for the pathogenesis of neurological melioidosis. mBio 5: e00025-14.

4. Kristensson K, 2011. Microbes' roadmap to neurons. Nat Rev Neurosci 12: 345-357.

5. Woods ML, Currie BJ, Howard DM, Tierney A, Watson A, Anstey NM, Philpott J, Asche V, Withnall K, 1992. Neurological melioidosis: seven cases from the northern territory of Australia. Clin Infect Dis 15: 163-169.
6. Arif MA, Abid MH, Renganathan R, Siddiqui KA, 2015. Central and peripheral nervous system involvement in neuromelioidosis. BMJ Case Rep 2015: bcr2015211001.

7. Vestal ML, Wong EB, Milner DA, Jr., Gormley WB, Dunn IF, 2013. Cerebral melioidosis for the first time in the western hemisphere. J Neurosurg 119: 1591-1595.

8. Kumar GSS, Raj PM, Chacko G, Lalitha MK, Chacko AG, Rajshekhar V, 2008. Cranial melioidosis presenting as a mass lesion or osteomyelitis. J Neurosurg 108: 243-247.

9. Feige J, Moser T, Hauer L, Pikija S, Sellner J, 2020. Clinical challenges in a 49-year-old patient with severe tick-borne myeloradiculitis despite complete active vaccination. Vaccines 8: 93.

10. Nardone R, Versace V, Brigo F, Tezzon F, Zuccoli G, Pikija S, Hauer L, Sellner J, 2017. Herpes simplex virus type 2 myelitis: case report and review of the literature. Front Neurol 8: 199.

11. Chetchotisakd P et al., 2014. Trimethoprim-sulfamethoxazole versus trimethoprim-sulfamethoxazole plus doxycycline as oral eradicative treatment for melioidosis (MERTH): a multicentre, double-blind, non-inferiority, randomised controlled trial. Lancet 383: 807-814.

12. Currie BJ, Fisher DA, Howard DM, Burrow JNC, Selvanayagam S, Snelling PL, Anstey NM, Mayo MJ, 2000. The epidemiology of melioidosis in Australia and Papua New Guinea. Acta Tropica 74: 121-127.

13. McLeod C, Morris PS, Bauert PA, Kilburn CJ, Ward LM, Baird RW, Currie BJ, 2015. Clinical presentation and medical management of melioidosis in children: a 24-year prospective study in the northern territory of Australia and review of the literature. Clin Infect Dis 60: 21-26.

14. Saravu K, Kadavigere R, Shastry AB, Pai R, Mukhopadhyay C, 2015. Neurologic melioidosis presented as encephalomyelitis and subdural collection in two male labourers in India. $J$ Infect Dev Ctries 9: 1289-1293.

15. Susilawathi NM, Darwinata AE, Dwija IB, Budayanti NS, Wirasandhi GA, Subrata K, Susilarini NK, Sudewi RAA, Wignall FS, Mahardika GNK, 2012. Epidemiological and clinical features of human rabies cases in Bali 2008-2010. BMC Infect Dis 12: 81.

16. Hsu CC, Singh D, Kwan G, Deuble M, Aquilina C, Korah I, Norton $R, 2016$. Neuromelioidosis: craniospinal MRI findings in Burkholderia pseudomallei infection. J Neuroimaging 26: 75-82.

17. Yata S, Ogawa T, Sugihara S, Matsusue E, Fujii S, Kinoshita T, 2004. HTLV-I carrier with unusual brain MR imaging findings. Neuroradiology 46: 755-758.

18. Konagaya M, lida M, 1991. A case of HTLV-1 associated myelopathy with diffuse white matter lesion of the frontal lobe and continuous lesion of the pyramidal tract on cranial MRI. Rinsho Shinkeigaku 31: 875-877.

19. Hildenbrand P, Craven DE, Jones R, Nemeskal P, 2009. Lyme neuroborreliosis: manifestations of a rapidly emerging zoonosis. AJNR Am J Neuroradiol 30: 1079-1087.

20. Rupprecht TA, Koedel U, Fingerle V, Pfister HW, 2008. The pathogenesis of lyme neuroborreliosis: from infection to inflammation. Mol Med 14: 205-212.

21. Anastasina M, Domanska A, Palm K, Butcher S, 2017. Human picornaviruses associated with neurological diseases and their neutralization by antibodies. J Gen Virol 98: 1145-1158.

22. Pruvost-Robieux E, Yeung J, Sudacevschi V, Cordoliani Y, De Malherbe M, Pico F, 2016. Reversible corticospinal tract hyperintensities in neurologic Lyme disease. Neurology 87: 548-549.

23. Conde Cardona G, Quintana Pájaro LD, Quintero Marzola ID, Ramos Villegas Y, Moscote Salazar LR, 2020. Neurotropism of SARS-CoV 2: mechanisms and manifestations. J Neurol Sci 412: 116824. 